\title{
Post-unification priorities for probation: former practitioners' perspectives
}

Two former practitioners who recently moved to academia reflect on their experiences of working in probation and identify some priorities for the service post-unification. 
As two recent former practitioners who are now working as lecturers on the Community Justice programme, we would like to use this article to reflect on our own experiences of Transforming Rehabilitation (TR) and unification. The impact of TR was deep, traumatic and hit hard, dividing staff both physically and professionally. This emotional impact led to experienced practitioners leaving the profession earlier than planned and has left the organisation lacking in experienced staff. Unification is a step in the right direction but will be a long process. For staff to be confident that any positive changes will remain in place for decades rather than a few years this transformation needs a long-term commitment from policy makers and leaders.

The Probation Workforce Strategy under the Target Operating Model (TOM) (HMPPS, 2021) sets out a positive picture in terms of providing staff with manageable workloads to allow the time for learning and development. There is also recognition that practitioners have endured substantial change in recent years because of TR. Implementation of the TOM commenced on 26 June 2021 but will take a long time to embed. It is worth noting that the 'TOM- roadmap' goes to April 2024 and beyond. The 'lift and shift' approach (HMIP, 2021) will mean that staff having a blended caseload will be deferred. It may well be the case that staff may struggle to cope with managing their existing caseloads and simultaneously complete any training required. Challenges in developing and refreshing the skills needed to work with a blended caseload will further be affected by practitioners continuing to work from home (due to the coronavirus (Covid-19) pandemic), as this will reduce the opportunities to collaborate and reflect with others in their team.

Staff will be feeling vulnerable and anxious, and do not necessarily share the enthusiasm regarding unification that is evident in the higher ranks of the Probation Service. This needs to

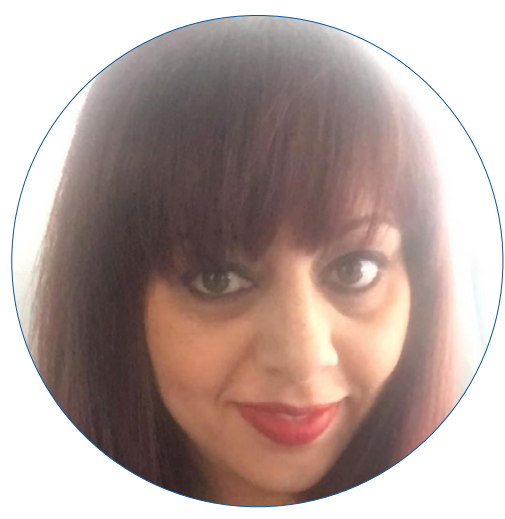

Deena Parmar

Lecturer in Community Justice De Montfort University

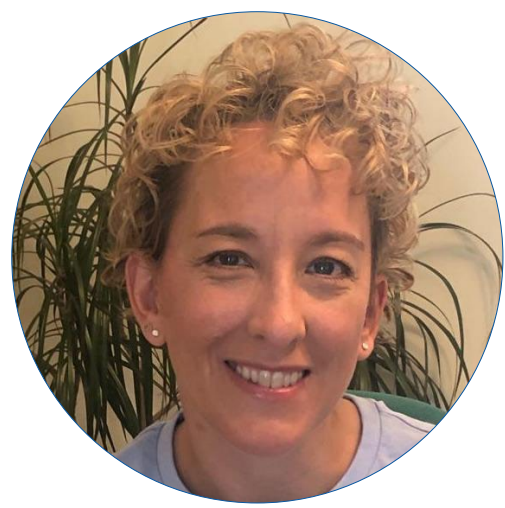

Daniella Nudd

Lecturer in Community Justice De Montfort University

be considered in the early stages of unification. 'Welcome events' need to be laying the foundations for an environment that encourages communication and acknowledge the mixed emotions that staff are experiencing. Centring on the importance of targets, updating risk registers and case records risks alienating some staff. How staff feel will always impact on their performance and this needs to be recognised and accounted for. 
Emotional literacy defines the skills that practitioners may use in understanding their own emotions and working effectively and appropriately with the emotions of offenders, victims and witnesses (Knight, 2014). Phillips et al (2020) highlight the importance of using, managing and displaying emotions when working with people on probation as critical to effective probation practice. The volume of information received by practitioners, often cascaded via email communication daily, risks impacting on morale and people's ability to remain emotionally literate in their work with people on probation. It is therefore important that Senior Managers are also emotionally literate having the ability to empathise and have insight into how practitioners are feeling. It is all too easy to compile and click 'send' on yet another email - achieving that sense of 'ticking the box' - without recognising how the email is received or whether the practitioner has read it (in their ever-overloaded inbox).

A further challenge for the organisation will be to align the differing skills between NPS and CRC practitioners and for both sets of practitioners to familiarise themselves with certain types of cases that they will not have worked with for some years. Having qualified in 2002, Deena Parmar was a Probation Officer in the CRC for four years. She then joined the NPS in 2018 and reflects that at the time she felt as though she had joined a completely different organisation. It took her approximately 12 months before feeling competent in completing an OASys that met the NPS Quality Assurance Standards and the same amount of time again to feel confident in working with people who posed a High Risk of Serious Harm, MAPPA cases and sex offender cases. It is by drawing upon these personal experiences that we anticipate challenges for CRC practitioners as unification embeds in its early stages. At the same time, former NPS Probation Practitioners will also need to (re)learn how to manage specific medium risk of harm cases without MAPPA oversight. As an example, CRC
Probation Practitioners have become accustomed to managing (often) chaotic and complex medium risk of harm domestic violence perpetrators, without the safety net of formal multi-agency arrangements in place to support them in managing risk.

In 2020 the NPS introduced the four pillars approach to risk management which incorporates the elements of supervision, monitoring and control, interventions and treatment and victim safety planning as developed by Kemshall (HMIP, 2020). Practitioners who have worked in the CRCs, will have either had a seven-year gap in working with sex offenders and holding MAPPA cases or have never had any experience of working with such caseloads. Therefore, (re) developing the skills needed for an OASys which will meet the standards expected within the Probation Service could well be a significant challenge for some practitioners. This will require time to embed and will need enabling and supportive line management to allay any undue pressure and anxiety.

There has recently been an injection of funding for the Probation Service, helping to more than double the recruitment of Probation Officers (GOV UK, 2021). In doing so, the organisation may start to be able to operate in a manner that allows for that collaborative and meaningful relationship between the practitioner and the person on probation, which is vital in terms of desistance. The impact of such an intensive recruitment drive will take time, and newly qualified Probation Officers will require that time to develop and improve their practice incrementally. The skills of these newly qualified practitioners will be different to those of the previous cohorts trained prior to Covid-19. This is due to the current cohorts having completed their training predominantly whilst working from home during the pandemic and under the Exceptional Delivery Model (EDM). 
The lack of consistent 'soft learning' in an office environment may have impacted on their skills and development, and the consequences of this could be felt for years to come. In terms of long-term challenges, this risks producing a generation of Probation Officers who are limited in some areas, potentially running in parallel to experienced staff leaving because of the emotional toll of yet more upheaval.

Whilst it is positive that HMPPS is recruiting more probation trainees to address staffing shortages, there is some concern that in some areas, trainees will outnumber experienced Probation Practitioners (HMIP, 2021). This could contribute to burnout due to less support being available to trainees in terms of both development of their practice and opportunities to reflect with experienced practitioners. In response to this, the organisation needs to put provisions in place to enhance the immediate post qualification period for newly recruited Probation Officers. This would support the development of their resilience and ensure that they are afforded the opportunity to consolidate their learning incrementally to guard against exiting the organisation prematurely. The celebratory mood to mark unification is of course welcomed, but it needs to be acknowledged that practitioners are tired of reorganisation. In our experience, staff want to make a difference for the people that they supervise. In order to do this, the Probation Service must consider the emotional impact of further change on staff, allow practitioners time to process the learning required to work with a blended caseload and recognise that the substantial recruitment of trainee Probation Officers is not an immediate solution to a long-term problem. Unification is an important milestone in the evolution of the Probation Service and a step in the right direction to deliver better outcomes for the public and the people who we supervise. Real transformation is, however, a long-term commitment, and unification is just the beginning of that journey (Russell cited in HMIP, 2021).

\section{References}

HMIP (2020) Risk of Serious Harm Guidance 2020 [Online] Available at: https://assets.publishing. service.gov.uk/government/uploads/system/uploads/ attachment data/file/897166/rosh-guidance-2020. pdf

HMIP (2021) A thematic review of work to prepare for the unification of probation services. [Online] Available at: https://www.justiceinspectorates.gov.uk/ hmiprobation/inspections/unification-of-probationservices/

HMIP (2021) Probation unification is not enough, by itself, to put right the flaws of past reform. [Online] Available at: https://www.justiceinspectorates.gov. uk/hmiprobation/media/press-releases/2021/06/ probation-unification-is-not-enough-by-itself-to-putright-the-flaws-of-past-reform/

HMPPS (2021) The Target Operating Model for probation services in England and Wales: Probation Reform Programme.[Online] Available at: https:// assets.publishing.service.gov.uk/government/uploads/ system/uploads/attachment data/file/959745/ HMPPS - The Target Operating Model for the Future of Probation Services in England Wales - English - 09-02-2021.pdf

Knight, C (2014) Emotional Literacy in Criminal Justice; Professional Practice with Offenders. Palgrave Macmillan

Phillips J, Westaby C and Fowler, A (2020) Emotional Labour in Probation. [Online] Available at: https:// www.justiceinspectorates.gov.uk/hmiprobation/wpcontent/uploads/sites/5/2020/04/Emotional-Labourin-Probation.pdf

Press release by GOV.UK (2021) Bigger, better Probation Service to cut crime. [Online] Available at: https://www.gov.uk/government/news/bigger-betterprobation-service-to-cut-crime

Probation Service (2020) Welcome Hub. [Online] Available at: https://welcome-hub.hmppsintranet.org.uk 